\title{
Preparations for p-Au run in 2015
}

\author{
C. Liu
}

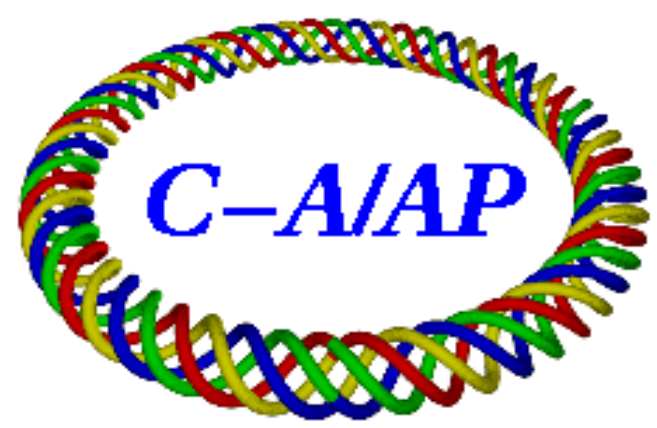

\section{Collider-Accelerator Department Brookhaven National Laboratory Upton, NY 11973}

\section{U.S. Department of Energy Office of Science, Office of Nuclear Physics}

Notice: This document has been authorized by employees of Brookhaven Science Associates, LLC under Contract No. DE-AC02-98CH10886 with the U.S. Department of Energy. The United States Government retains a nonexclusive, paid-up, irrevocable, world-wide license to publish or reproduce the published form of this document, or allow others to do so, for United States Government purposes. 


\title{
Preparations for p-Au run in 2015
}

\author{
C. Liu, Brookhaven National Lab, Upton, NY, U.S.A.
}

December 31, 2014

\section{Introduction}

Although RHIC has provided collisions of asymmetrical species in past years, the p-Au run in 2015 will be unique in this different species collision category. This is resulted from the different charge mass ratio of the proton and fully stripped $\mathrm{Au}$ ion (1 vs. 79/197). The p-Au run requires a special acceleration ramp, and movement of a number of beam components as required by the beam trajectories. The DX magnets will be moved for the first time in the history of RHIC. In this note, the planning and preparations for $\mathrm{p}-\mathrm{Au}$ run will be presented. For more details, please refer to the run planning wiki page [1].

\section{Acceleration ramp}

During the d-Au run in 2003, long range beam-beam interactions resulting from unequal revolution frequencies of the two beams caused significant beam loss on the first half of the ramp [2, 3. The strategy of accelerating beams to top energies with their revolution frequencies being equal has since been adopted. For the p-Au run, one can not raise the injection energy of Au beam (to beam rigidity of $196 \mathrm{Tm}$ ) to match the beam frequencies because of the limit of the injection kicker strength (90 Tm in term of beam rigidity). It is not practical either to lower the injection energy of the proton beam (to beam rigidity of 33 $\mathrm{Tm}$ ) otherwise it crosses transition during acceleration. The mini-ramp scheme was proposed by M. Blaskiewicz in which the Au beam will be injected first, then accelerated crossing transition to $\gamma=25.4$. After that the proton beam will be injected and both beams will be accelerated to $100 \mathrm{GeV} /$ nucleon with matched revolution frequencies. This scheme was tested with Au beam only in 2014 by C. Montag [4, to check the possible instability of the $\mathrm{Au}$ beam sitting just above transition energy for $\sim 10$ minutes for proton beam injection. The test was successful since only expected horizontal emittance growth was observed due to intra-beam scattering. 


\section{Movement of components required by $\mathrm{p}-\mathrm{Au}$ trajectories}

With equal revolution frequencies and roughly equal circumferences of the proton and $\mathrm{Au}$ beam, the beam rigidities are quite different (at store, $518 \mathrm{Tm}$ for proton and $832 \mathrm{Tm}$ for Au beam). The horizontal bending angles in the common DX magnets are therefore different for two beams. Both beams would be at angles with respect to the line across the DX centers. The beam trajectories [5] at colliding IRs (6 and 8) and non-colliding IRs (2, 4, 10 and 12) at store are shown respectively in Figs. 1 and 2. Crossing angles were in the design of beam trajectories for all IRs at injection to alleviate the aperture limits at DX magnets. The trajectories of the beams demand the movements of a number of beam components. In 2015, RHIC run will start with 9 weeks of proton proton collision, followed by 5 weeks of p-Au run and 2 weeks of $\mathrm{p}-\mathrm{Al}$ run. It is desirable to move components before pp for quick and smooth transition between pp and p-Au programs, without impact on the pp run.

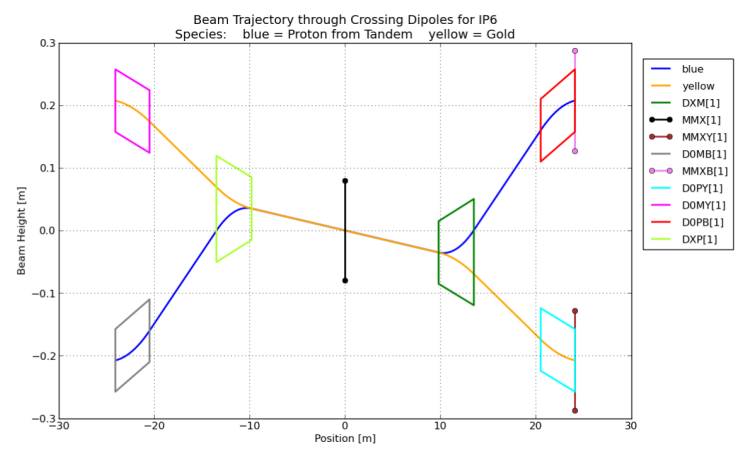

Figure 1: Beam trajectories at colliding IRs (6 and 8) at store.

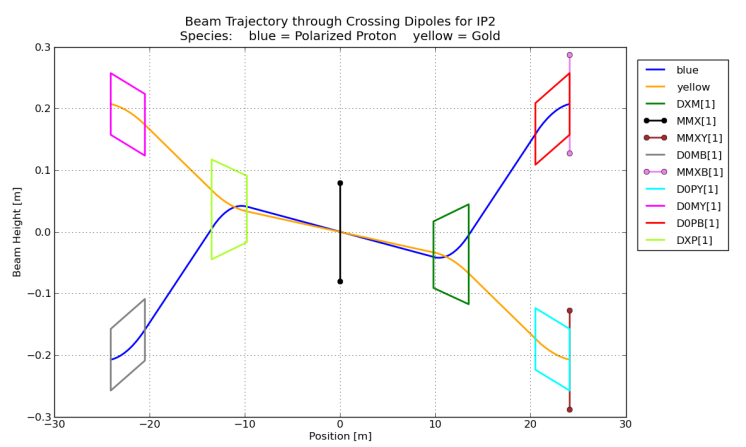

Figure 2: Beam trajectories at non-colliding IRs (2, 4, 10 and 12) at store. 


\subsection{DX magnets}

Both DX aperture scan study and orbit data from He3-Au run indicate the beams can be off-center by $47 \mathrm{~mm}$ at DX edge (13.5 m from IPs) at injection, $50 \mathrm{~mm}$ at store without noticeable beam loss. The beam trajectories of $\mathrm{p}-\mathrm{Au}$ were designed based on these findings. The amount by which the DX magnets need to be moved are thereafter determined. The amounts and directions of DX movements are listed in Table1. The moving directions are always towards the Yellow beamline. It reduces the available physical apertures at DX magnets for pp run if they got moved. The counter measure is to design beam trajectories with crossing angle at the corresponding IRs, which would not result in circumference difference. Simulation indicates that there is no effect on beam polarization from crossing angle at IRs 11. Therefore, IR2 and 4 DX magnets were moved before pp run. Proton beam with non-zero crossing angle would not be desirable at IR10 and 12 because of the electron lens device at IR10 and $\mathrm{H}$-jet at IR12. The same is true at colliding IRs (6 and 8 ) because experiments want head-on collision with zero common angle. Therefore, IR6, 8, 10, and 12 DX magnets will be moved between pp and p-Au run. IR2 and 4 DX magnets

Table 1: DX magnets movement specifications

\begin{tabular}{cccc}
\hline When & Sector & Distance $(\mathbf{m m})$ & Direction \\
\hline \multirow{3}{*}{ Before pp run } & 1 & 20 & outwards \\
& 2 & 20 & inwards \\
& 3 & 17.5 & inwards \\
& 4 & 17.5 & outwards \\
\hline \multirow{3}{*}{ Between pp and p-Au run } & 5 & 25 & outwards \\
& 6 & 25 & inwards \\
& 7 & 25 & inwards \\
& 9 & 25 & outwards \\
& 10 & 20 & outwards \\
& 11 & 20 & inwards \\
& 12 & 20 & inwards \\
& & &
\end{tabular}

were moved during shutdown since no effect on proton polarization for pp run was observed in simulation. As this report is written, all other DX magnets except those at sector 6,7 and 8 have been moved and put back for testing.

\section{2 $9 \mathrm{MHz}$ cavity}

Between the $9 \mathrm{MHz}$ cavity and the sector $4 \mathrm{DX}$ magnet, there was no space for a new bellow which would take $\sim 20 \mathrm{~mm}$ position shift. So decision was made to rotate the cavity so that one end (close to DX) moves together with DX magnet while the other end (close to IP4) remains fixed in position. Moving DX magnet by a larger amount works better for the $\mathrm{p}-\mathrm{Au}$ trajectories, however 
imposes aperture limits for beam trajectories in pp run. The compromise was made to move IR3 and 4 DXs by $17.5 \mathrm{~mm}$. The existing bellow in IR3 was replaced by a new one with more flexibility. The $9 \mathrm{MHz}$ cavity and DX magnets will all be moved back after 2015 run.

\subsection{Roman pots}

During the shutdown, new vertical Roman pots, which reside in DX-D0 chambers, were installed in sector 5 and 6 . The beam position relative to the Roman pots detector centers are listed in Table 2. For p-Au run, only sector 6 side Ro-

Table 2: Beam positions relative to Roman pots centers

\begin{tabular}{ccc}
\hline RP & $\begin{array}{c}\text { pp run } \\
\text { Distance }(\mathbf{m m})\end{array}$ & $\begin{array}{c}\text { p-Au run } \\
\text { Distance }(\mathbf{m m})\end{array}$ \\
\hline sector 5 DX side RP & 1.9 & 24.1 \\
sector 5 D0 side RP & 22.2 & 3.3 \\
sector 6 DX side RP & 1.9 & 27.4 \\
sector 6 D0 side RP & 22.2 & 40.6 \\
\hline
\end{tabular}

man pots will be used to detect scattered proton beam. The decision was made to move the sector 6 DX-D0 chamber laterally inwards by $25 \mathrm{~mm}$ (maximum limited by the bellows) to maximize the acceptance of Roman pots.

\subsection{ZDCs}

Beams will collide head-on in both experiments, however, both beam have a common angle with respect to the line across DX centers. The forward neutrons will be at an angle of $3.61 \mathrm{mrad}$. The physical size of the ZDCs are $\pm 5 \mathrm{~cm}$ in horizontal plane. Therefore, the decision was made to move sector 5 and 8 DX-D0 chamber laterally by $25 \mathrm{~mm}$ so that ZDCs can be moved to intercept forward neutrons.

\subsection{DX-D0 chambers}

Beam trajectories in DX-D0 chambers were also studied for possible aperture limit. The beam trajectories were added to the DX-D0 chamber drawings by S. Nayak for the study. The design trajectories are closest to the chamber wall in the middle of the DX-D0 chamber (see Table 3).

The numbers in parentheses are for injection energy. The spaces between beam and the vacuum wall translate to at least 7 times the rms beam size. Besides movement of DX-D0 chambers at sector 5, 6 and 8 for ZDCs and Roman pots, only sector 7 DX-D0 chamber will be rotated so its DX end moves with DX magnet. Further beam tests are planned to justify not to move other DX-D0 chambers in non-colliding IRs. 
Table 3: Distance from beams to the wall in DX-D0 chambers

\begin{tabular}{c|cc|cc|c}
\hline & \multicolumn{2}{|c|}{ Minimum gap between } & \multicolumn{2}{c|}{ Minimum gap between } & Distance \\
& \multicolumn{2}{|c|}{ Blue beam and wall $(\mathrm{mm})$} & \multicolumn{2}{c}{ Yellow beam and wall $(\mathrm{mm})$} & prom IP $(\mathrm{m})$ \\
\hline & pp run & p-Au run & pp run & p-Au run & \\
\hline sector 5/6 & 46.5 & $28.6(22.4)$ & 46.5 & $64.8(63.8)$ & 17.7 \\
sector $7 / 8$ & 48.5 & 30.0 & 48.5 & 43.9 & 17.51 \\
sector 3/4 & 48.5 & 27.7 & 48.5 & 58.9 & 17.7 \\
other sectors & 48.5 & 25.9 & 48.5 & 73.7 & 17.7 \\
\hline
\end{tabular}

\subsection{STAR beam pipe}

In $2014 \mathrm{He} 3-\mathrm{Au}$ run, there was background hitting at STAR detector when injecting Helium beam in the Blue ring. In the same year, STAR internal target experiment observed unexpected signal in horizontal plane. These brought on heated discussion during the preparation of p-Au run. During the FY14/15 shutdown, the interior of beam pipe from DX to DX in IR6 was examined visually using a boroscope. Loose RF fingers were found in both sides of IP6, however in vertical plane. These were fixed. Then a survey was done for beam pipe in IR6 before the tie-in of the experiment magnet. The sector 5 end of the Be pipe was found off by $\sim 7 \mathrm{~mm}$ horizontally inbound, which explain the two said mysteries. The beam pipe was realigned afterwards. A similar survey was done in PHENIX as well. No significant offset was found.

\subsection{AC dipole}

The inner radius of the AC dipole is $20 \mathrm{~mm}$. With a $4.18 \mathrm{mrad}$ beam angle at IR4, the beam would be off-center by $15 \mathrm{~mm}$ at the AC dipole located $3.53 \mathrm{~m}$ away from IP4, which is certainly an aperture limit concern. Therefore, the AC dipole was taken out temporarily for $\mathrm{p}-\mathrm{Au}$ run. This was done before the $\mathrm{pp}$ run to save time later when transitioning between the pp and p-Au run.

\section{References}

[1] Reports and documents regarding p-Au run, http://www.cadops.bnl. gov/AGS/Operations/OpsWiki/index.php/FY15_Run_Planning_To_Do_ List\#References

[2] T. Satogata, L. Ahrens, M. Bai, J. Beebe-Wang, M. Blaskiewicz, J.M. Brennan, K.A. Brown, D. Bruno, P. Cameron, J. Cardona, R. Connolly, A. Drees, W. Fischer, R.P. Fliller III, G. Ganetis, C. Gardner, J.W. Glenn, H. Hahn, T. Hayes, H. Huang, U. Iriso-Ariz, W.W. MacKay, A. Marusic, R. Michnoff, C. Montag, F. Pilat, V. Ptitsyn, T. Roser, K. Smith, S. Tepikian, and D. Trbojevic, "Commissioning of the RHIC deuteron-gold collisions", 
proceedings of the 2003 Particle Accelerator Conference, Portland, Oregon, BNL-69612-CP (2003).

[3] W. Fischer, P. Cameron, S. Peggs, and T. Satogata, "Tune modulation from beam-beam interaction and unequal radio frequencies in RHIC", in "Beam halo dynamics, diagnostics, and collimation", proceedings of HALO'03 and Beam-Beam'03, Montauk, New York, AIP Conference Proceedings 693 (2003).

[4] C.Montag, M. Blaskiewicz, V. Schoefer, I. Blackler, A. Marusic, T. Shrey, "p-Au Ramp Test", C-AD tech note, C-A/AP/518

[5] S. Tepikian, D. Trbojevic, "DX magnet requirements for p-Au operation", C-AD tech note, $\mathrm{C}-\mathrm{A} / \mathrm{AP} / 447$ 